\title{
3D Controlled Motion of a Microrobot using Magnetic Gradients
}

\author{
Karim Belharet, David Folio and Antoine Ferreira \\ Institut PRISME, ENSI de Bourges, 88 boulevard Lahitolle, 18020 Bourges, FRANCE; \\ \{karim.belharet, david.folio, antoine.ferreira\} @ensi-bourges.fr
}

\begin{abstract}
This paper presents an endovascular navigation of a ferromagnetic microdevice using MRI-based predictive control. The concept was studied for future development of microrobot designed to perform minimally invasive interventions in remote sites accessible through the human cardiovascular system. A system software architecture is presented illustrating the different software modules to allow 3D navigation of a microdevice in blood vessels, namely: (i) vessel path extraction, (ii) magnetic gradient steering, (iii) tracking and (iv) closed-loop navigation control. First, the navigation path of the microrobot into the blood vessel is extracted using Fast Marching Method from the pre-operation images (3D MRI imaging) to guide the microrobot from the injection point to the tumor area through the anarchic vessel network. Based on the pre-computed path, a Model Predictive Controller is proposed for robust time-multiplexed navigation along a $3 \mathrm{D}$ path in presence of pulsative flow. The simulation results suggest the validation of the proposed image processing and control algorithms.
\end{abstract}

keywords: Microrobot, gradient controlled motion, endovascular navigation

\section{INTRODUCTION}

Microrobots designed to perform targeted therapy by navigating in the cardiovascular system are a prolific research area for minimally invasive surgeries [1][2] and treatments efficiency through early diagnosis of diseases [3]. When microrobots are propelled in the body fluids, especially in the blood circulatory system, a very large number of remote locations in the human body become accessible. Because the method of propulsion should allow such a microrobot to navigate through the cardiovascular system, the use of the normal blood flow itself must be considered only as a complementary means of propulsion when the travel path is along the direction of the blood flow. These untethered microrobots have been mainly developed according to three different designs: magnetic bead pulling [2], biomimetic flagellated robot [4] and magnetotactic bacteria [5]. Furthermore, navigation requires observation of the scene in order either to plan the trajectory by off-line mapping, or to correct on-line the microrobot's pose error between the planned and the observed trajectory. Recently, magnetic resonance imaging (MRI)-based medical microrobotic platforms are investigated to reach locations deep in the human body while enhancing targeting efficacy using real-time navigational and trajectory control [6]. For the position recognition of the microrobot in the blood vessels, from the pre-operation images, 3D path planning and route optimization 
solutions have been proposed. The authors in [7] proposed an endovascular path-planning method based on 3D potential fields and enhanced breath-first search algorithms based on MR-imaging. Based on these path-planning techniques, only explorative 2D control strategies have been adopted so far using simple proportional-integralderivative (PID) controller [8]. However, stability and robustness are not ensured against important perturbations. First, pulsatile flow should be taken into account since variations in waveform, amplitude, and frequency exists from vessels. Second, variation of time-multiplexed sequence parameters (duty cycle of the propulsion gradients, and repetition time of the tracking sequence) produce important trajectory errors during real-time navigation. Finally, random imaging signal noise degrades the localization of the microrobot during tracking.

The main objective of this paper is to propose an automated technique based on image processing and control algorithms for path finding, reconstruction and navigation control of a ferromagnetic microrobot using the MRI system. The MRI-based control of a ferromagnetic microcapsule presented here is dedicated to macroscale navigation, which focuses in conveying a microsphere radius of $300 \mu \mathrm{m}$ in vessels, such as arteries and arterioles (eg, around 10 to $0.5 \mathrm{~mm}$ ), as close as possible to the tumor area. Based on slice images provided by an MRI system, relevant information related to detection of blood vessels is extracted using robust Frangi vesselness filtering from the pre-operation images. Then, a set of minimal path is predefined, using Fast Marching Method (FMM), to guide the ferromagnetic microrobot from the injection point to a targeted area through the anarchic vessel network. Based on the precomputed path, a Model Predictive Controller (MPC) is designed. Hence, we proposed a control strategy for robust time-multiplexed navigation along a $3 \mathrm{D}$ path even in presence of pulsative flow. The simulation results suggest the validation of the proposed image processing and control algorithms against perturbations.

\section{MRI-based NAVIGATION SYSTEM}

\subsection{System Overview}

The Magnetic Resonant Imaging (MRI)-based microrobotic system is used here for propulsion and navigation of the microdevice. The propulsion of the ferromagnetic microcapsule in the cardiovascular system is realized through the induction of force from magnetic gradients provided by the MRI. This MRI system will guide the microcapsule in vivo through vascular networks to a targeted area. The overview of the software system architecture is given in Fig. 1.

(i) The graphical user interface module, which comprises input command prompt, 3D-visualization, and process supervision tools. (ii) The control module, which comprises (a) the high-level controller responsible for the microcapsule navigation tasks and for the generation of the magnetic field gradients and (b) the low-level controller (manufacturer MRI controller) responsible for the generation of the desired field gradients and for the image acquisition tasks. Then, (iii) the controlled hardware, which comprises (a) the MRI hardware and software systems and (b) the microcapsules that have been injected within the vasculature and are steered by the magnetic gradients. Finally, (iv) the image processing module, which comprises the MRI data rendering, the navigation path extraction, and the microdevice recognition/tracking algorithm. In particular, the tracking 
procedure, presented in [9], allows to estimate the position and accumulation of the microdevices within the vasculature, the tissues, and the organs of the human. However as MR-imaging is too slow to gather position of the microrobot and special MR-tracking algorithm, such as MR-SET, must considered to ensure real time control.

\subsection{Navigation in blood vessels}

In our context, the problem of navigation in blood vessels within the MRI data can be formulated as finding the correct way through the data that follows the vessel of interest between its start and end points. Finding a navigation path within the endovascular network is then an essential, primary, and important step that must be addressed prior to the control procedure. The problem of vessels extraction has received considerable attention in the computer vision and medical imaging communities [10]. Hence, several class of methods have been proposed to find a path from a set of medical imaging, such as using tracking methods [11] [6] [12], path extraction methods [13][14][15], and so on. Most works based on in vivo MR-tracking methods usually need many user-defined way points as the input of a controller module for the navigation computation. A major drawback in general remains when the user must define many points (e.g. way or fiducial points) manually. Hence, for a complex structure (e.g. colon, small vessels...) the required interactivity can be very tedious. The path extraction is useful for a range of application domains including medical image analysis, robot navigation, and artificial intelligence. The path extraction technique needs a very simple initialisation and leads to global minimum of a snake-like energy, thus avoiding local minima. Moreover, it is fast and accurate. This path finding problem has been studied for ages by mathematicians, and has been solved numerically using graph theory or dynamic programming. Cohen and Kimmel [16] solved the minimal path problem in 2D with a front propagation equation between the two fixed end points, using the Eikonal equation (that physically models wave-light propagation), with a given initial front. Wink et al.[14] explored different methods to determine the minimum cost path through a pre-defined cost image, for extraction of vessel centrelines from medical image data. Among them are Dijikstra's algorithm, the $A^{\star}$ algorithm, which makes use of additional heuristics to steer the search process, and wave front propagation analysis [15]. Early, Sethian [17] explored the use of Fast Marching Method (FMM) to extract minimal paths. This method relies on the fact that the gradient of the FMM arrival function has only one local minimum, which is guaranteed to be global minimum [13]. Therefore, the minimal path can be extracted by back-propagating from given seeds (e.g. the end point of the desired path) to the starting point implicitly embedded in the arrival function.

In this work the FMM is adopted to design a set of paths to guide the microdevice from the injection point to the tumor area through the vessel network. Our aim is to focus on the automation of the path construction, reducing the need of interaction and improving performance, in a robust way. 


\section{MRI-BASED PREDICTIVE CONTROL}

\subsection{Problem formulation}

Navigation in blood vessel requires observation of the scene in order either to plan the path by offline mapping, or to correct online the microrobot pose error between the planned and the observed path. To insure a smooth conveyance of the microcapsule to destination, collisions and the risk to be trapped by the endothelium, optimal navigation performance will be affected by external perturbations and MRI technological constraints:

- Nonnegligible pulsatile flow, whose variations in waveform, amplitude, and frequency exist from one vessel to another (such as arteries and arterioles).

- Magnetic gradients are used both for observation and control purposes in a time-multiplexed sequence. It requires different trade-offs in terms of refresh rate, duty cycle of the propulsion gradients, and repetition time of the tracking sequence.

- MRI overheating avoidance leading to limitations on the MRI duty cycle, tends to increase the disproportional scaling between magnetic forces used for control purpose and perturbation forces (drag forces and net buoyancy forces).

- Limitations on the magnetic gradient amplitude in available MRI devices.

- Proper delay in the image processing algorithms that renders the navigation control unstable.

\subsection{Real-Time Sequence Design}

The overall concept of the in-vivo MRI-tracking system is based on the fact that both tracking and propulsion is possible with the gradient coils of the MRI system. Software based upgrading of a clinical MRI system is the least expensive approach to convert a platform that is used for imaging to an effective interventional platform. At any instant only one of the functions could be applied (i.e. either tracking or propulsion), but both will be executed over the same MRI interface. The MRI interface has therefore to be shared and a time-division-multiple-access scheme for it has to be developed. Fig. 2 shows an overview of the real-time sequence with time-multiplexed positioning and propulsion phases introduced by Martel et al. [6]. The main aspect relevant to the controller's performance is (i) the duty cycle $T_{\text {Prop }} / T_{\mathrm{S}}$ that stands for the ratio between the propulsion time and the time between two successive position requests, and (ii) the synchronization event delay $T_{\text {Sync }}$ that stands for the minimum time allowed for image processing and real-time control feedback. First, the duty cycle should be adapted to apply sufficient magnetic propulsion gradients during a predefined propulsion time $T_{\text {Prop }}$ to prevent the microrobot from drifting away from the path. Second, a large time delay $T_{\text {Sync }}$ produces oscillations as the microrobot approaches the reference path $w$ leading to position instabilities. Such limitations have been pointed out by Mathieu et al. [8] when implementing simple proportional-integral-derivative (PID) controller. We proposed a Model Predictive Controller (MPC) including microrobot's motion and dynamics with estimation of the pulsative blood flow and time-multiplexed positioning. A predictive path-tracking control consider a prediction window (cf. Fig. 2). The 
propulsion phase starts during $T_{\text {Prop }}$ seconds at the same initial condition as the prediction phase, recording the performance of the system according to a prediction horizon. After this phase the system ends after a imagingpropulsion sequence at a final position $Y$ which is set as the new initial condition of the next prediction output $\widehat{Y}$. The proposed navigation based predictive controller offers stability by design and allows the designer to tradeoff performance for (computation) speed, stability margins according to the MRI application and technological requirements outlined in section 3.1.

\subsection{Model description}

The linear model used in this work is derived from the nonlinear model developed in a previous study [18]. The authors used this model to combine the backstepping controller and high-gain observer in order to control the path of the microrobot inside a vessel using the MRI gradients.

The different forces acting on the microrobot are (see Fig. 3): drag force $\vec{F}_{d}$, apparent weight $\vec{W}_{a}$ and magnetic force $\vec{F}_{m}$. The application of Newton's third law and the projection on the $(\vec{x}, \vec{y}, \vec{z})$ axes leads to:

$$
\left\{\begin{aligned}
m \ddot{x} & =\vec{F}_{d x}+\vec{F}_{m x} \\
m \ddot{y} & =\vec{F}_{d y}+\vec{F}_{m y} \\
m \ddot{z} & =\vec{F}_{d z}+\vec{F}_{m z}+\vec{W}_{a}
\end{aligned}\right.
$$

where $m$ is the mass of the microrobot modeled by a ferromagnetic sphere with a high saturation magnetization.

Let $\overrightarrow{\mathbf{v}}=\left(v_{f_{x}}, v_{f_{y}}, v_{f_{z}}\right)$ denotes the blood flow velocity, and $(x, y, z)$ the robot location in the blood vessel wrt. to a given frame $\mathscr{F}(O, \vec{x}, \vec{y}, \vec{z})$. Taking the drag coefficient $C_{d}=\frac{24}{R e}$, the linear model can be written as follow:

$$
\left\{\begin{array}{l}
\ddot{x}=\alpha_{1}\left(\dot{x}-v_{f_{x}}\right)+\alpha_{2} u_{x} \\
\ddot{y}=\beta_{1}\left(\dot{y}-v_{f_{y}}\right)+\beta_{2} u_{y} \\
\ddot{z}=\gamma_{1}\left(\dot{z}-v_{f_{z}}\right)+\gamma_{2} u_{z}
\end{array}\right.
$$

with the following parameters $\alpha_{i}, \beta_{i}$ and $\gamma_{i}$, and the magnetic gradients considered as control inputs $u_{x}, u_{y}$ and $u_{z}$, that is:

$$
\left\{\begin{aligned}
\alpha_{1} & =-4.5 \frac{\eta \cos \varphi \cos \theta}{r^{2} \rho}, & u_{x} & =\left\|\vec{\nabla} B_{x}\right\| \\
\beta_{1} & =-4.5 \frac{\eta \cos \varphi \sin \theta}{r^{2} \rho}, & & u_{y}=\left\|\vec{\nabla} B_{y}\right\| \\
\gamma_{1} & =-4.5 \frac{\eta \sin \varphi}{r^{2} \rho}, & & u_{z}=\left\|\vec{\nabla} B_{z}\right\| \\
\alpha_{1} & =\beta_{2}=\gamma_{2}=\frac{m}{\rho} & &
\end{aligned}\right.
$$

where $\rho$ is the density of the fluid; $\eta$ is the fluid viscosity; $r$ is the spherical radius of the microrobot; and $\mathbf{B}=\left(B_{x}, B_{y}, B_{z}\right)^{T}$ is the magnetic field generated by the MRI system.

Finally, the state-space representation is deduced from (2):

$$
(S)\left\{\begin{aligned}
\dot{x} & =v_{x} \\
\dot{v_{x}} & =\alpha_{1} v_{x}-\alpha_{1} v_{f_{x}}+\alpha_{2} u_{x} \\
\dot{y} & =v_{y} \\
\dot{v_{y}} & =\beta_{1} v_{y}-\beta_{1} v_{f_{y}}+\beta_{2} u_{y} \\
\dot{z} & =v_{z} \\
\dot{v_{z}} & =\gamma_{1} v_{z}-\beta_{1} v_{f_{z}}+\gamma_{2} u_{z}
\end{aligned}\right\}
$$


where $\left(v_{x}, v_{y}, v_{z}\right)^{T}$ denotes the robot velocity along $\vec{x}$-axis, $\vec{y}$-axis and $\vec{z}$-axis. Assuming that the microrobot location $(x, y, z)$ can be measured thanks to the MRI system, we denote by $Y=(x, y, z)^{T}$ the process measure. We can notice that system $(S)$ can be divided into three subsystems $\left(S_{x}\right),\left(S_{y}\right)$ and $\left(S_{z}\right)$, which allow us to define three independents MPC schemes to track the reference path $w$ in 3D MRI data.

In this paper, we aim to use the system model defined in (4) in a high level MPC scheme in order to follow efficiently a pre-planed path extracted with the method proposed in section 2.2. The proposed MPC controller is intended to embed the low level robust controller designed in [18][19] (see Fig. 4). Let us notice that the low level controller provides ${ }^{1}$ robustness performances against MRI system constraints (as pulsatile flow, limited gradient amplitude, and image noise) while the MPC controller is designed to tradeoff time-multiplexed sequence constraints in terms of refresh-rate, duty cycle of the propulsion gradients, and repetition time of the tracking sequence.

\subsection{Predictive Control}

Model Predictive Control (MPC) has become an area of significant research interest over the last past twenty years. This interest has been powered by a stream of successful industrial applications [21]. When focusing on linear (and unconstrained) discrete time transfer function models and quadratic cost functions, some of the best known approaches include the Generalized Predictive Control (GPC) introduced by Clarck et al.[22], and the inner loop stabilizing Stable Predictive Control [23].

MPC refers to a class of computer control algorithms which use an explicit process model to predict the future response of the system. One way to design MPC is to use an extended state-space representation, which is given by:

$$
\begin{cases}\dot{X}_{k+1} & =A X_{k}+B \Delta u_{k} \\ Y_{k} & =C X_{k}\end{cases}
$$

where $\Delta u_{k}=u_{k}-u_{k-1}$ is the discrete difference operator. The predicted state vector at time $k+i$ is then computed from [24]:

$$
\widehat{X}_{k+i \mid k}=A^{i} X_{k \mid k-1}+\sum_{j=0}^{i-1} A^{j} B u_{k-j-1}
$$

Then, the future outputs $Y_{k+j \mid k}$ can be computed based on the plant for future times starting at time $k$ using a recursion procedure, defined by:

$$
\widehat{Y}_{k+i \mid k}=C\left(A^{i} X_{k}+\sum_{j=1}^{i} A^{i-j-1} B u_{k+i-j \mid k}\right)
$$

Design criterion is defined for certain interval of predictions (several steps to future). It includes the part of control error, in which the model of system is covered (insertion of equations of prediction (7)) and the part of control actions, where the input energy (control actions) is weighted. This part redistributes control errors to individual steps of predictions and provides coupling within intervals of predictions. Usual form of the criterion

\footnotetext{
${ }^{1}$ The interested reader is invited to refer to [18][19][20].
} 
for predictive design is written as follows:

$$
J_{k}=\sum_{j=N_{1}+1}^{N_{2}}\left[Y_{k+j} W_{k+j}^{T} Q_{Y} Y_{k+j} W_{k+j}\right]+\sum_{j=1}^{N_{u}}\left[u_{k+j-1}^{T} Q_{u} u_{k+j-1}\right]
$$

The criterion is expressed in step $k . N=N_{2}-N_{1}$ is a horizon of prediction, $N_{u}$ is the control horizon. $Q_{Y}$ and $Q_{u}$ are output and input penalizations. $Y_{k+j}$ and $u_{k+j-1}$ are output and input (full or incremental) values.

Finally, let us note how to construct real control actions at incremental algorithm: after computing a vector for whole horizon, only first control $u_{k}$ is used; then to obtain the full control actions the second line of equation (5) is applied.

When using MPC in the state-space formulation, and generally at the use of whichever stat-space control, it is necessary to solve the question of availability of the state of the system (state vector). If it is not available, and only system output from the measurement are known, then some state-space estimation has to be considered. Suitable well-known solution of such estimation is the state-space observer based on Kalman filter.

\section{Results}

\subsection{Navigation Path Extraction}

The FMM algorithm, introduced by Sethian [17] is applied here to extract a targeted navigation path within the vessel network. From the set of 3D MRI data, a speed map is computed (ie. a weighting image map) in order to enhance the relevant intravascular network. Choosing an appropriate and efficient image cost function is the most difficult part of the entire process.

We describe in the sequel presented in Fig. 5, the process used to extract the navigation path. First, we need a relevant cost function which allow to enhance vessel in the image. To this aim, we used some a priori knowledge about vessel shape and intensity in MRI data (cf. Fig. 5). Vessels are expected to appear as bright tubular structures in a darker environment. One way to account for the varying size of vessels is by multiscale analysis. It allows us to detect structures of different sizes according to the scale at which they give maximal response. In this context, a typical speed image is produced by using a Frangi vesselness filter [25] which uses the eigenvectors of the Hessian matrix at each voxel of the image to compute the likeliness of an image region to vessels. This mapping is selected in such a way that vessels regions will have higher speed (high level in speed image, see Fig. 5). Once the speed map is generated, the user has to select a start and end points (ie. seed points) in the viewer of the input original image. The FFM will then propagate a front from the start seed and traveling to the targeted area, thanks to the speed map. Thus, this approach allow to find a minimal navigation path between the start and targeted seed (cf. Fig. 5).

\subsection{Microdevice Navigation using Predictive Control}

Simulations conducted here are performed within the scope of actual commonly spread MRI system abilities. Actually, MRI systems are able to generate magnetic gradients with an intensity of some tens of $\mathrm{mT} . \mathrm{m}^{-1}$. Let us note that this limitation is additionally affected by the gradient coils duty-cycle and by the time-multiplexing 
sequence $\left(T_{\text {Prop }} / T_{\mathrm{S}}\right)$ needed both for controlling and observing sequences. In order to make sure that the amplitude of the control inputs remains bounded by physical actuators limits $u_{i, \max }$, and to protect the system, we applied a simple time-scaling factor defined by $\frac{u_{i}}{k(t)}$, with $k(t)=\max \left\{1, \frac{u_{i}}{u_{i, \max }}\right\}$. The following set of simulations corresponds to a microcapsule radius of $r=300 \mu \mathrm{m}$ and a time scaling settled at $k(t)=0.55$. Different situations are considered to illustrate and to validate the performance of the proposed MRI-based predictive controller.

We validated the proposed control strategy on a 3D endovascular navigation path $w$ extracted from MRI-data with the previously presented method without any disturbance. Then, to evaluate the efficiency of the proposed MRI-based predictive controller, we performed some tests with a white Gaussian noise on the system output measure $Y$. The noise is about $10 \%$ of the measured signal which is motivated by the common precision of clinical MRI devices.

Fig. 6 describes the navigation path followed by the microrobot, and Fig. 7 presents the relative error norm between the current position $Y$ and the reference $w$, for a time horizon $N=5$. As shown, the system output $Y$ follows perfectly the reference path $w$. In particular, the microdevice is able to reach quickly the navigation path, in spite of a gap between the initial position $Y$ and the reference $w$. Let us notice that the 3D tracking is not too much affected by the Gaussian noise since position standard deviation (STD) and root mean square (RMS) error are quite satisfactory.

Fig. 8 simulates the impact of the time horizon $N$ on the system tracking error for optimized time horizon values between $3 \leq \mathrm{N} \leq 10$. Comparing the error statistics, the nature of anticipation of the MPC scheme is well illustrated - important values of $N$ improve greatly the anticipation nature of the path behavior but increases at the same time the path tracking error-. Hence, a great value of $N$ does not necessarily guarantee good performances, and classically increase the complexity of the scheme.

To evaluate the robustness of our control strategies, we have also applied an external disturbance which represent the pulsatile blood flow behavior.

Hence, we have modeled the blow flow as a time-sinusoidal profile with spatial parabolic form $\sigma_{\text {flow }}(t)$. In case of an artery, to model the action of flow (positive) and reflux of blood (negative) $\sigma_{\text {flow }}(t)$ were shifted up, leading to:

$$
\sigma_{\text {flow }}(t)=0.025\left(1+1.15 \sin \left(2 \pi t+\frac{\pi}{8}\right)\right)
$$

where amplitude of 0.025 is representative of maximum blood flow speed. In the following, the performance and stability of the predictive controller with respect to physiological parameters variations are demonstrated in Fig. 9. It appears that the system is robust to uncertain physiological parameters (disturbance applied at $t=13$ seconds) since the $3 \mathrm{D}$ position tracking error norm is bounded to $0.2 \%$.

Finally, we have evaluated the robustness of our controller for different duty cycle values: $25 \%, 50 \%$ and $75 \%$, respectively. The error curves and statistics for the three scenario are illustrated on Fig. 10. As one can see for a duty cycle of $50 \%$, that is the MRI system propels during the first half-period, and acquires 3D images on the other half-period, the path tracking is quite satisfactory. It corresponds to the real-time navigational and trajectory control constraints reported in [8]. Nevertheless for a duty cycle of $25 \%$ (ie. the MRI propels during $1 / 4$ period, and image during $3 / 4$ period) the microrobot can not properly track the reference path $w$ leading 
to great tracking error. The time-multiplexing duty cycle is optimized for a value around $40 \%$. In the optimal case, the simulated predictive behavior of the MPC controller suggests better tracking results compared to those obtained by [8] that should be confirmed by experimentation.

\section{CONCLUSIONS}

The proposed MRI-based ferromagnetic microcapsule steering and navigation strategy has been developed at milli and microscale, where endovascular navigation path extraction and predictive controller have been designed. The main drawback of MRI-based navigation stems from the strong limitations on the magnetic gradient amplitude of available MRI devices. As magnetic forces used for propelling are volumetric, whereas the drag force is at best dependent on the microcapsule's area, the smaller the capsule, the higher the required control forces with respect to hydrodynamic perturbations. Consequently, this approach is well conditioned for beads whose radius is up to a few dozen micrometers with actual MRI devices. Targeting aims at focusing these microcarriers and stopping them through embolization at the arterioles entry close to the occluded blood vessels. Possible releasing mechanisms could rely on biodegradable polymer and techniques used in hyperthermia where aggregates of nanocapsules can be heated to melt polymer. Such a solution is actually under experimentation for validation of the proposed minimally invasive MRI-based microrobotic system.

\section{ACKNOWLEDGMENTS}

This work was supported by European Union's 7th Framework Program and its research area ICT-2007.3.6 Micro/nanosystems under the project NANOMA (Nano-Actuactors and Nano-Sensors for Medical Applications). The PhD student K. Belharet is supported by the Nano-IRM project, funded by Conseil Gnral du Cher and Agglomeration de Bourges.

\section{References}

[1] K. Ishiyama, M. Sendoh, and K. I. Arai, "Magnetic micromachines for medical applications," J. of Magnetism and Magnetic Materials, vol. 242-245, pp. 41-46, 2002.

[2] J. Abbott, Z. Nagy, F. Beyeler, and B. Nelson, "Robotics in the Small," IEEE Robot. Automat. Mag., p. 92, 2007.

[3] T. A. Cavalcanti, B. Shirinzadeh and S. Ikeda, "Nanorobot for Brain Aneurysm,” Int. J. of Robotic Research, vol. 28, no. 4, pp. 558-570, 2009.

[4] B. Behkam and M. Sitti, "Design methodology for biomimetic propulsion of miniature swimming robots," ASME J. Dyn. Syst. Meas. Control, pp. 36-43, 2006. 
[5] S. Martel, M. Mohammadi, O. Felfoul, Z. Lu, and P. Pouponneau, "Flagellated magnetotactic bacteria as controlled MRItrackable propulsion and steering systems for medical nanorobots operating in the human microvasculature," Int. J. Robot. Res., vol. 28, no. 4, pp. 571-582, 2009.

[6] O. Felfoul, J. Mathieu, G. Beaudoin, and S. Martel, "In vivo MR-tracking based on magnetic signature selective excitation," IEEE Trans. Med. Imag., vol. 27, no. 1, pp. 28-35, Jan. 2008.

[7] W. Sabra, M. Khousam, and S. Martel, "Use of 3D potential field and an enhanced breath-first search algorithms for path-planning of microdevices propelled in the cardiovascular system," in 27th IEEE EMBS Annual Int. Conf., Shangai, China, 2005, pp. $91-100$.

[8] S. Tamaz, A. Chanu, J.-B. Mathieu, R. Gourdeau, and S. Martel, "Real-time mri-based control of a ferromagnetic core for endovascular navigation," IEEE Trans. Bio-Med. Eng., vol. 55, no. 7, pp. 1854-1863, July 2008.

[9] T. Wortmann, C. Dahmen, and S. Fatikow, "Study of MRI susceptibility artifacts for nanomedical applications," Journal of Nanotechnology in Engineering and Medicine, vol. 1, no. 4, p. 041002, 2010.

[10] C. Kirbas and F. Quek, "A review of vessel extraction techniques and algorithms," ACM Comput. Surv., vol. 36, no. 2, pp. 81-121, 2004.

[11] P. Reuzé, J. Coatrieux, L. Luo, and J. Dillenseger, “A 3D moment based approach for blood vessel detection and quantification in MRA," Technology and Health Care, vol. 1, pp. 181-188, 1993.

[12] J. Tokuda, G. S. Fischer, S. P. DiMaio, D. G. Gobbi, C. Csoma, P. W. Mewes, G. Fichtinger, C. M. Tempany, and N. Hata, "Integrated navigation and control software system for mri-guided robotic prostate interventions," Computerized Medical Imaging and Graphics, vol. 34, no. 1, pp. 3-8, 2010.

[13] T. Deschamps, "Curve and shape extraction with minimal path and level-sets techniques: Applications to 3d medical imaging," Ph.D. dissertation, University of Paris Dauphine, 2001.

[14] O. Wink, W. J. Niessen, and M. A. Viergever, "Minimum cost path determination using a simple heuristic function," Int. Conf. on Pattern Recognition, vol. 3, p. 7010, 2000.

[15] O. Wink, W. Niessen, B. Verdonck, and M. Viergever, "Vessel axis determination using wave front propagation analysis," Medical Image Computing and Computer-Assisted Intervention, vol. 2208, pp. 845-853, 2001, lecture Notes in Computer Science.

[16] L. Cohen and R. Kimmel, "Global minimum for active contour models: A minimal path approach," Int. J. of Computer Vision, vol. 24, no. 1, pp. 57-78, 1997.

[17] J. A. Sethian, Level set methods and fast marching methods. Cambridge University Press, 1999.

[18] L. Arcèse, M. Fruchard, and A. Ferreira, "Nonlinear modeling and robust controller-observer for a magnetic microrobot in a fluidic environment using MRI gradients," in IEEE/RSJ Int. Conf. on Intel. Robots and Systems, St. Louis, MO, USA, Oct. 2009, pp. 534-539. 
[19] L. Arcese, A. Cherry, M. Fruchard, and A. Ferreira, "High gain observer for backstepping control of a mriguided therapeutic microrobot in blood vessels," in IEEE/RAS-EMBS Int. Conf. on Biomedical Robotics and Biomechatronics, Tokyo, Japan, Sep. 2010, pp. 349 -354.

[20] L. Arcese, A. Cherry, M. Fruchard, and A. Ferreira, "Dynamic behavior investigation for trajectory control of a microrobot in blood vessels," in IEEE/RSJ Int. Conf. on Intel. Robots and Systems, Taipei, Taiwan, Oct. 2010, pp. $5774-5779$.

[21] S. Qin and T. Badgwell, "A survey of industrial model predictive control technology," Control engineering practice, vol. 11, no. 7, pp. 733-764, 2003.

[22] D. Clarke, C. Mohtadi, and P. Tuffs, "Generalized predictive control - Part I \& II," Automatica, vol. 23, pp. 137-160, 1987.

[23] B. Kouvaritakis, J. Rossiter, and A. Chang, "Stable generalised predictive control: an algorithm with guaranteed stability," IEE Proceedings Part D Control Theory and Applications, vol. 139, no. 4, pp. 349-362, 1992.

[24] E. Camacho and C. Bordons, Model predictive control. Springer Verlag, 2004.

[25] A. Frangi, W. Niessen, K. Vincken, and M. Viergever, "Multiscale vessel enhancement filtering," Lecture Notes in Computer Science, pp. 130-137, 1998. 


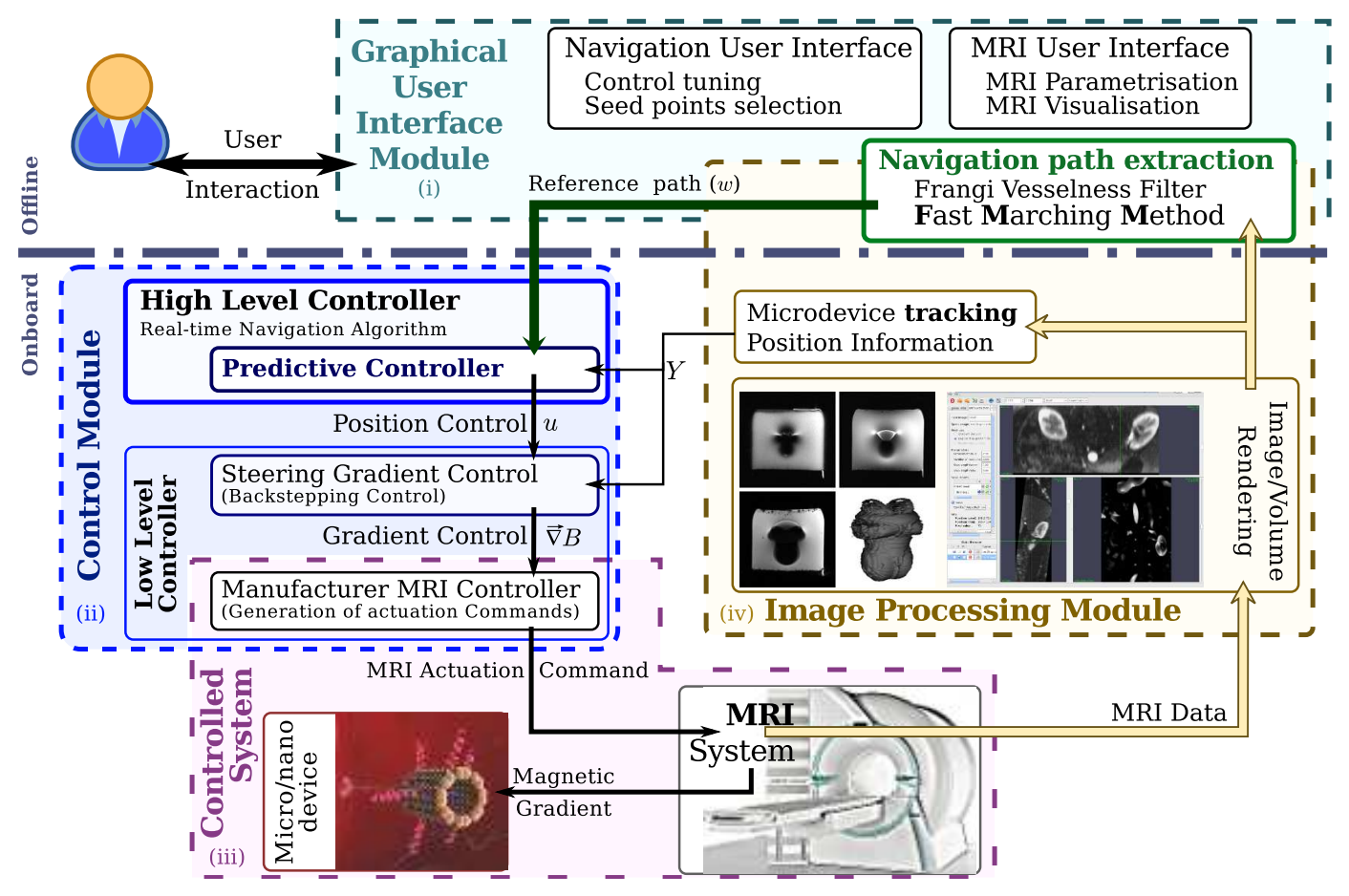

Figure 1: Overall system overview.

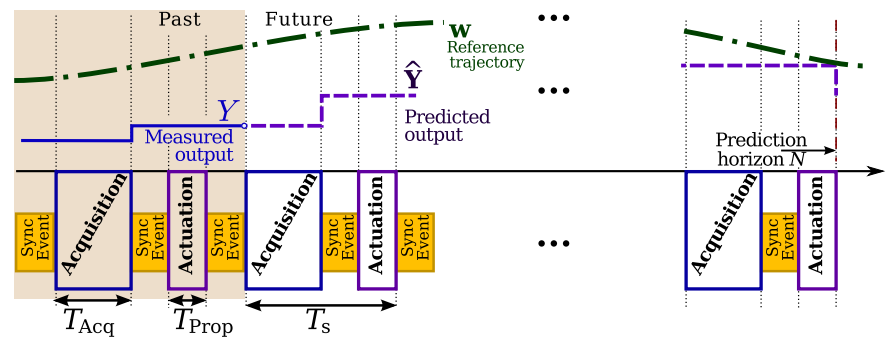

Figure 2: Timeline of acquisition and control prediction. 


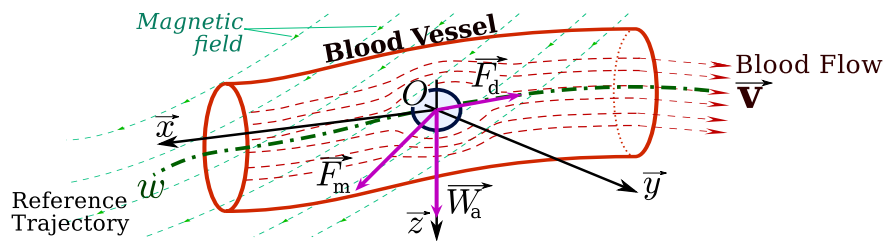

Figure 3: Forces applied on microrobot navigating in blood vessel.

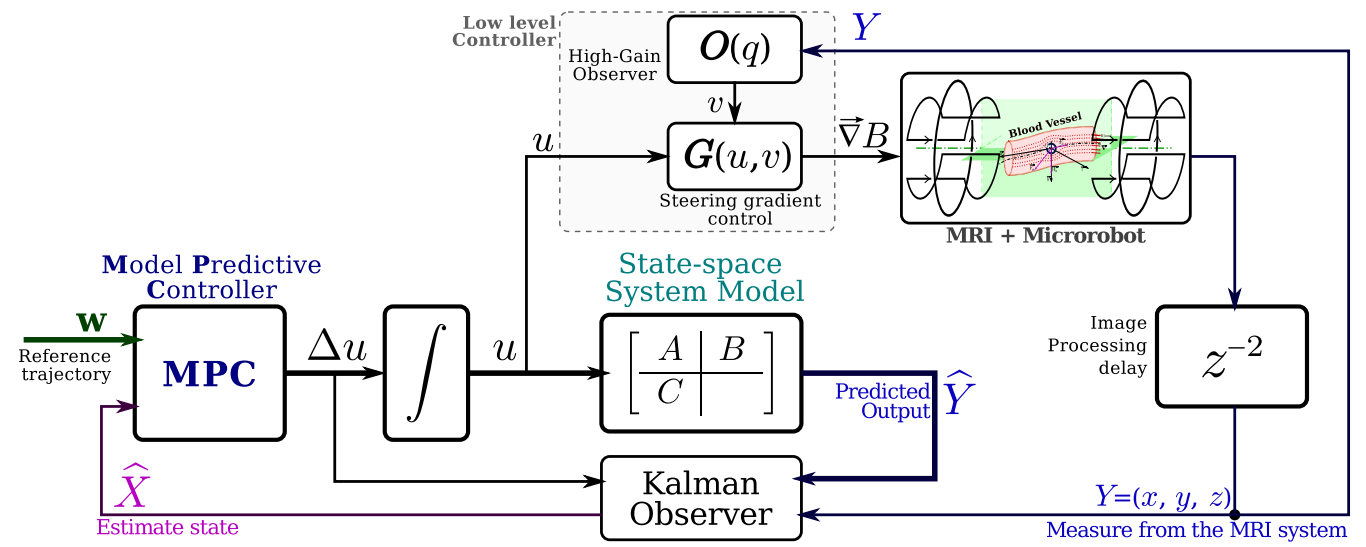

Figure 4: MRI-based Predictive Control strategy.

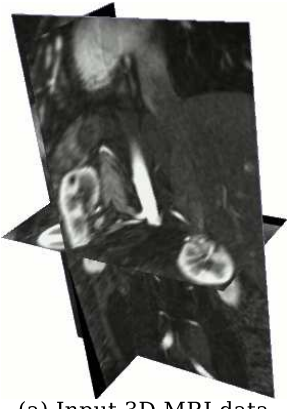

(a) Input 3D MRI data

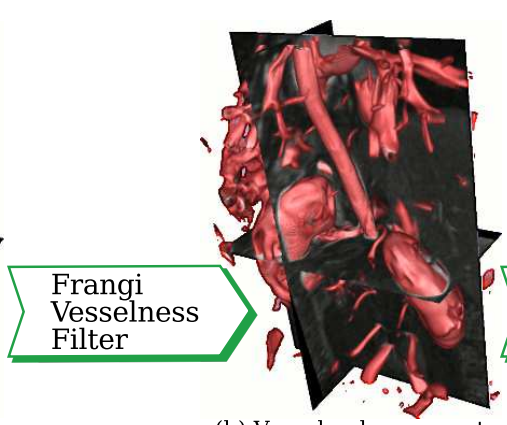

(b) Vessel enhancement

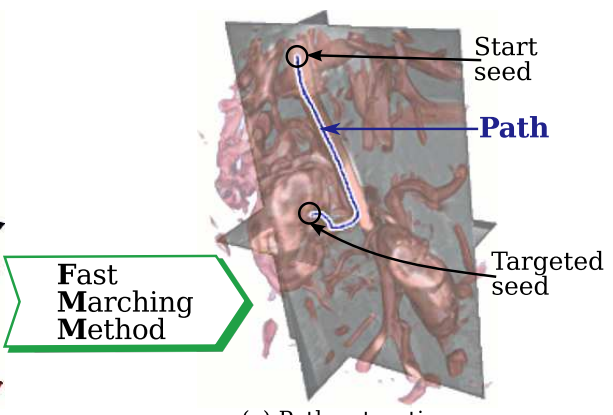

(c) Path extraction

Figure 5: 3D Navigation path extraction processing: a) Original MRI data; b) Computed cost function using Frangi filter (here displayed in red volume); c) Extracted navigation path between the start and end seeds using FFM. 


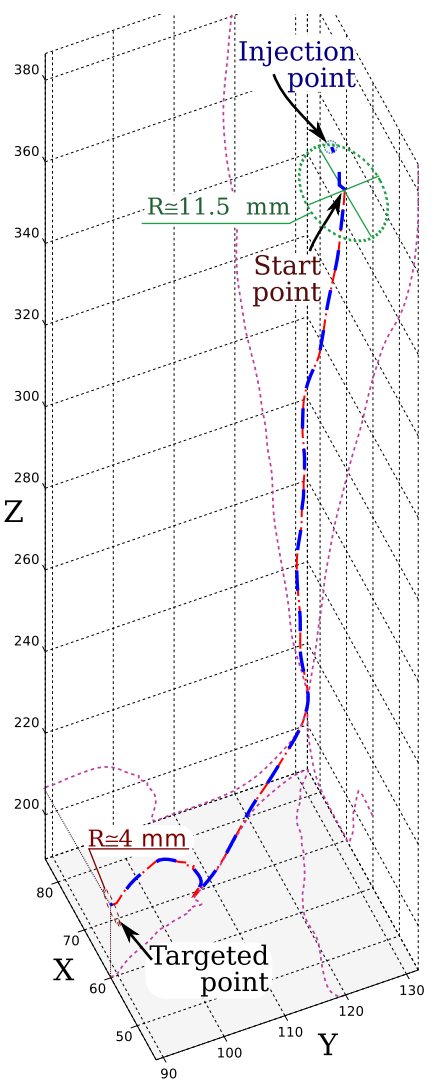

(a) 3D trajectories.

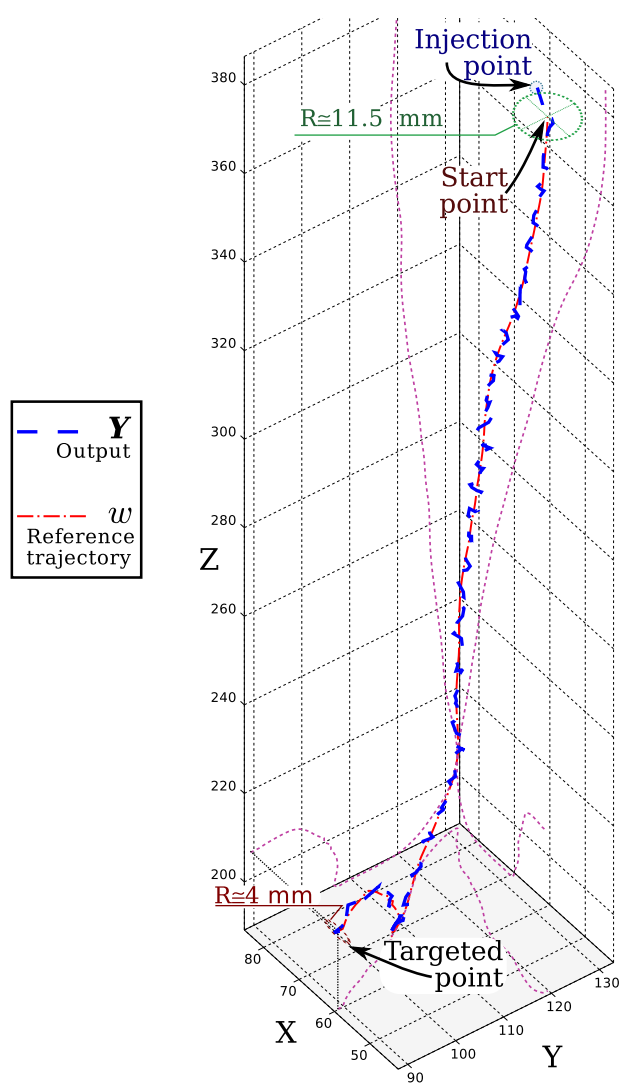

(b) with a white noise.

Figure 6: 3D MRI-based microrobot endovascular navigation $(N=5$, in $\mathrm{mm})$.

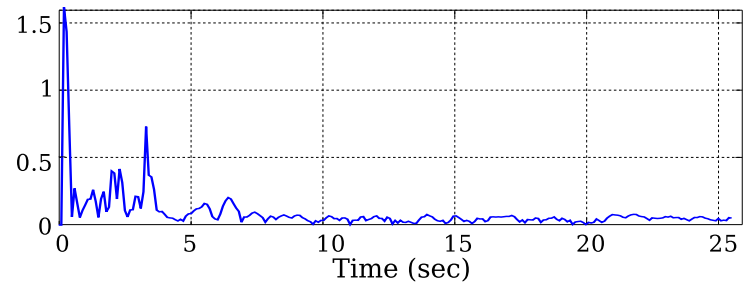

(a) Without noise

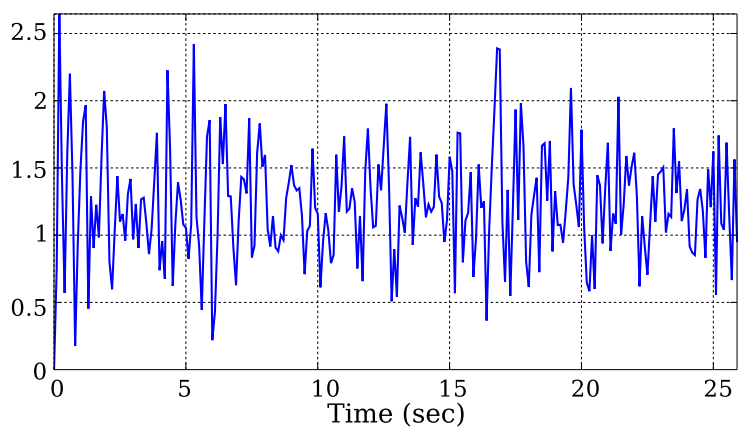

(b) With white noise

Figure 7: 3D tracking error: $\|Y-w\|$ for $N=5$. 


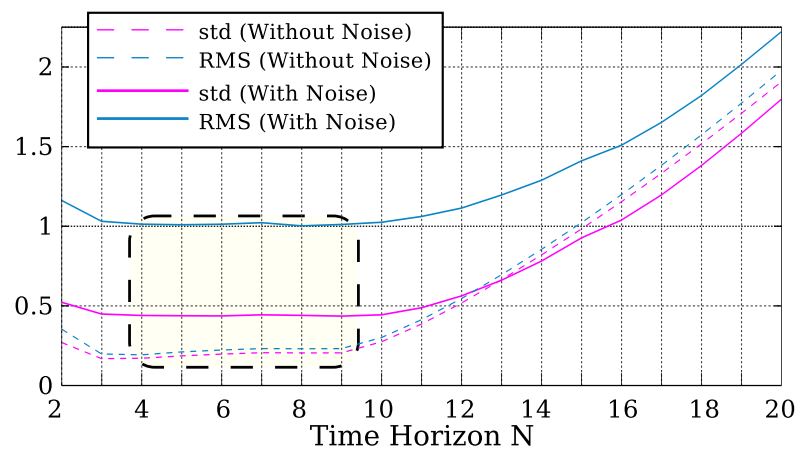

Figure 8: Tracking error statistics for different time horizons $N$.

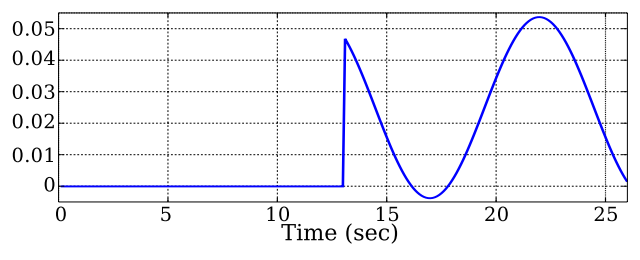

(a) Blood flow disturbance $\sigma_{\text {flow }}(t)$.

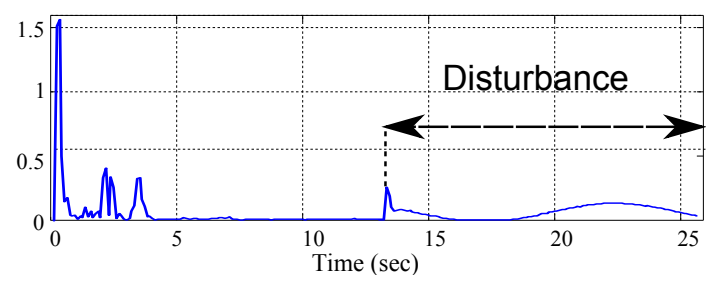

(b) Tracking error.

Figure 9: MRI-based navigation with blood flow disturbance.

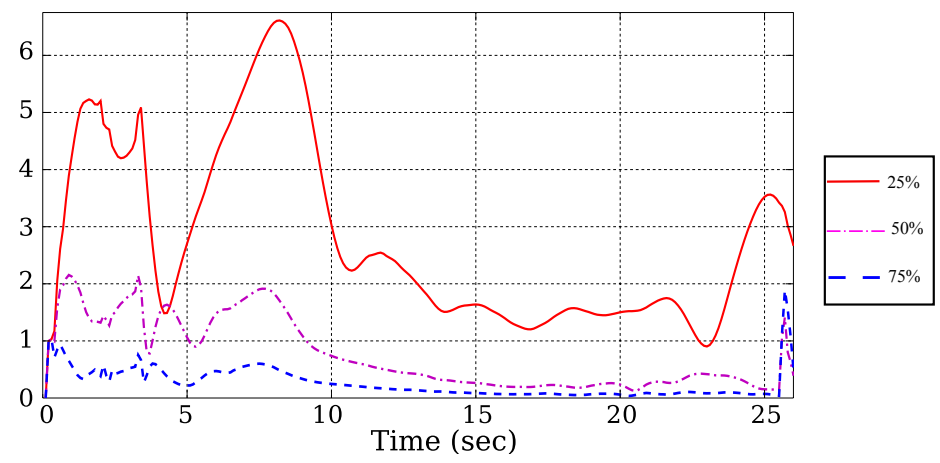

\begin{tabular}{|r|c|c|c|}
\hline & $25 \%$ & $50 \%$ & $75 \%$ \\
\hline \hline std & 1.5871 & 0.6860 & 0.2563 \\
\hline RMS & 3.2023 & 0.9697 & 0.3628 \\
\hline
\end{tabular}

Figure 10: Tracking error with different values of duty cycle. 